\title{
Consumo de drogas e conhecimento sobre suas consequências entre os estudantes de Odontologia
}

\author{
Drug consumption and knowledge about its consequences among students of Dentistry \\ Consumo de drogas y conocimiento de sus consecuencias en los estudiantes de Odontología
}

Recebido: 20/07/2021 | Revisado: 26/07/2021 | Aceito: 30/07/2021 | Publicado: 05/08/2021

Lukas Mendes de Abreu

ORCID: https://orcid.org/0000-0003-2791-3603 Universidade Estadual Paulista, Brasil

E-mail: lm.abreu@unesp.br

Luana de Lima Poroca Almeida

ORCID: https://orcid.org/0000-0001-7938-3283 Centro Universitário Tiradentes, Brasil

E-mail: luanaporoca@hotmail.com

Bergson Carvalho de Moraes

ORCID: https://orcid.org/0000-0002-8289-2510 Hospital Municipal Arthur Ribeiro Saboya, Brasil E-mail: bergsoncarvalho@gmail.com

Nilton José da Silva Filho

ORCID: https://orcid.org/0000-0001-5802-8362 Universidade Estadual Paulista, Brasil E-mail: niltonjfh@gmail.com

Ranielly de Souza Silva

ORCID: https://orcid.org/0000-0002-5844-7564 Centro Universitário Tiradentes, Brasil E-mail: ranielly.souza@souunit.com.br

João Artur Peixoto Granja

ORCID: https://orcid.org/0000-0001-8897-9396 Centro Universitário Tiradentes, Brasil

E-mail: artur.granja@hotmail.com

Lisandre Letícia Gomes da Silva

ORCID: https://orcid.org/0000-0003-4338-1598 Centro Universitário Tiradentes, Brasil

E-mail: lisandre_leticia2011@ @otmail.com João Wictor Leandro da Silva

ORCID: https://orcid.org/0000-0002-9941-147X Centro Universitário Tiradentes, Brasil E-mail: wictor_joao@hotmail.com

Renata Patrícia Freitas Soares de Jesus ORCID: https://orcid.org/0000-0002-7106-2554

Universidade Federal de Pernambuco, Brasil Centro Universitário Tiradentes, Brasil E-mail: rpfjesus@gmail.com

\begin{abstract}
Resumo
O consumo de drogas entre os universitários vem se elevando ao longo dos anos. O objetivo do estudo foi avaliar o consumo de drogas e o conhecimento sobre suas consequências entre estudantes de odontologia da região metropolitana do Recife. Trata-se de um estudo descritivo, transversal de abordagem quantitativa e qualitativa, com 210 estudantes, do primeiro e último período de instituições públicas e privadas de Pernambuco. Foram aplicados três questionários: um com os indicadores sociais, um para uso de drogas e outro sobre o conhecimento das consequências do uso de álcool, maconha e cocaína. Empregou-se estatística descritiva, Qui-quadrado de Pearson e o Teste t de Student para análise dos dados. A frequência do uso de drogas na vida foi de $81,90 \%$ para bebidas alcoólicas, $25,24 \%$ derivados de tabaco, 24,76\% maconha, 10,48\% inalantes, 7,14\% hipnóticos ou sedativos, 5,71\% alucinógenos, 4,76\% anfetaminas ou êxtase, 3,33\% cocaína e 1,43\% opióides. Quanto ao conhecimento das consequências, 96,67\% obtiveram mais da metade de acertos em relação ao álcool, $87,14 \%$ para maconha e 90,95\% para a cocaína. Há uma alta prevalência do consumo de drogas e um bom nível de conhecimento sobre as consequências do uso de álcool, maconha e cocaína entre os universitários.
\end{abstract}

Palavras-chave: Estudantes de odontologia; Drogas ilícitas; Alcoolismo. 


\begin{abstract}
Drug use within students has been rising over the years. The objective of this study was to evaluate drug consumption and the knowledge about its consequences among Odontology's student population in Recife and the Metropolitan area. This is a descriptive, cross-sectional study of a quantitative and qualitative approach, with 210 students of the first and last semesters of public and private schools in Pernambuco participated in the study. In this study, three questionnaires were used: one with the social indicators, another one for drug use, and the last one about knowing the consequences of using alcohol, marijuana and cocaine consumption. Statistic descriptive, Pearson Qui-square, and student T-test were used to analyze the data. It was observed that life time drug use was $81,90 \%$ of alcohol beverages, $25,24 \%$ tobacco products, $24,76 \%$ marijuana, 10,48\% inhalants, 7,14\% hypnotics or sedatives, 5,71\% hallucinogens, $4,76 \%$ amphetamines or ecstasy, 3,33\% cocaine e 1,43\% opioids. For the consequence's awareness, it was observed $96,67 \%$ obtained more than half of the correct answers regarding alcohol, 87,14\% for marijuana and $90,95 \%$ for cocaine. There is a high prevalence of drug use and a good level of knowledge about the consequences of alcohol, marijuana, cocaine use among universities students.
\end{abstract}

Keywords: Dental students; Illicit drugs; Alcoholism.

\title{
Resumen
}

El consumo de drogas entre los estudiantes universitarios se ha incrementado a lo largo de los años. El objetivo del estudio fue evaluar el uso de drogas y el conocimiento sobre sus consecuencias entre los estudiantes de odontología de la región metropolitana de Recife. Se trata de un estudio descriptivo, transversal, con enfoque cuantitativo y cualitativo, con 210 estudiantes, del primer y último período de instituciones públicas y privadas de Pernambuco. Se aplicaron tres cuestionarios: uno con indicadores sociales, uno para el consumo de drogas y otro sobre el conocimiento de las consecuencias del consumo de alcohol, marihuana y cocaína. Para el análisis de los datos se utilizó estadística descriptiva, chi-cuadrado de Pearson y prueba t de Student. La frecuencia de consumo de drogas en la vida fue $81,90 \%$ para bebidas alcohólicas, $25,24 \%$ derivados del tabaco, 24,76\% marihuana, 10,48\% inhalantes, 7,14\% hipnóticos o sedantes, 5,71\% alucinógenos, 4,76\% anfetaminas o éxtasis, 3,33\% cocaína y 1,43\% opioides . En cuanto al conocimiento de las consecuencias, el 96,67\% tuvo más de la mitad de respuestas correctas en relación al alcohol, el 87,14\% para la marihuana y el 90,95\% para la cocaína. Existe una alta prevalencia de consumo de drogas y un buen nivel de conocimiento sobre las consecuencias del consumo de alcohol, marihuana y cocaína entre los estudiantes universitarios.

Palabras clave: Estudiantes de odontología; Drogas ilícitas; Alcoholismo.

\section{Introdução}

O uso de drogas constitui um dos problemas de saúde pública mundial, considerando-se a magnitude e a diversidade de aspectos envolvidos. Esse consumo percorre diferentes países, contextos geográficos e culturais, classes sociais e faixas etárias; provoca prejuízos pessoais, familiares e sociais, alto custo econômico, assim como retroalimenta a violência urbana, familiar e interpessoal (Kerr-Corrêa, Andrade, Bassit \& Boccuto, 1999; Vargas \& Duarte, 2011).

Estimativas revelam que 201 a 341 milhões de pessoas, o equivalente a 5,5\% da população mundial, com idade entre 15-64 anos, fazem uso de drogas ilícitas, acarretando em cerca de 585.000 mil mortes, nos últimos anos. Além disso, em torno de 35 milhões de pessoas sofrem de transtornos decorrentes do uso de drogas e necessitam de tratamento. A prevenção e o tratamento continuam insuficientes em muitas partes do mundo, por ano, apenas uma em cada sete pessoas com transtornos decorrentes do uso indevido de drogas recebe tratamento (Castaldelli-Maia, et al., 2014).

No que concerne especificamente ao uso de drogas no Brasil, de acordo com o III Levantamento Nacional sobre o Uso de Drogas pela População Brasileira, o álcool é a substância mais consumida, onde a prevalência do uso de bebidas alcoólicas nos últimos 12 meses foi de 43,1\%, o que representa aproximadamente 65.943 milhões de habitantes. Além disso, os resultados revelaram que 3,2\% dos brasileiros usaram drogas ilícitas nos 12 meses anteriores à pesquisa, o que equivale a 4,9 milhões de pessoas. Esse percentual é muito maior entre os jovens nos quais cerca de 7,4\% das pessoas entre 18 e 24 anos haviam consumido drogas ilícitas no ano anterior à entrevista (Almeida, 2017). A população jovem apresenta as maiores taxas de uso de drogas e comportamento de risco, e cerca de $40 \%$ desta população é composta por universitários (Vieira, et al., 2008). Nesse contexto, é observado um aumento significativo no consumo de drogas entre os universitários (Eckschmidt, Andrade \& Oliveira, 2013; Mustafá, et al., 2019; Gonçalves, Fava, Alves \& Dázio, 2019; Mendonça, 2020).

Os estudantes da área de saúde merecem atenção especial quanto ao uso de drogas, pois é de fundamental importância 
o conhecimento do padrão de consumo e do conhecimento em relação às drogas que os futuros profissionais de saúde têm ou adquirem na formação acadêmica, devido ao efeito multiplicador de suas informações para a população assistida (Stempliuk, et al., 2005; Canoletti \& Soares, 2005; Silva, Malbergier, Stempliuk \& de Andrade, 2006). Em virtude disso, o presente estudo teve por objetivo avaliar o perfil do consumo de drogas e o conhecimento sobre suas consequências entre estudantes de Odontologia da região metropolitana do Recife.

\section{Metodologia}

Trata-se de um estudo descritivo, transversal de abordagem quantitativa e qualitativa (Estrela, 2018). O projeto foi previamente aprovado pelo Comitê de Ética em Pesquisa com seres humanos da Faculdade Integrada De Pernambuco FACIPE, sob o número do parecer 3.297.83. Compuseram a amostra 210 estudantes matriculados no primeiro e último períodos dos cursos de graduação em odontologia pertencentes a duas universidades públicas e duas particulares de Recife e região metropolitana.

Foram utilizados como critério de inclusão: alunos de ambos os sexos, efetivamente matriculados no primeiro ou décimo período do curso de odontologia, presente em sala de aula no período designado para a coleta dos dados e aqueles que declararam livremente interesse voluntário em participar da pesquisa. Os critérios de exclusão foram os alunos com idade inferior a 18 anos, efetivamente matriculado entre os segundo e nono períodos e os que não concordaram em participar da pesquisa.

Para coleta de dados foram utilizados três instrumentos autoaplicáveis, validados por experts e submetidos a testes pilotos a fim de adequá-los a realidade local. O primeiro tratou-se de um roteiro elaborado com base nos indicadores sociais mínimos descritos pelo Instituto Brasileiro de Geografia e Estatística (IBGE) com variáveis sociodemográficas de idade, sexo, raça, religião, situação conjugal, ocupação, com quem reside e semestre e turno do curso (Brasil, 2010).

Para avaliar o padrão de consumo de drogas foi utilizado o questionário Alcohol smoking and substance involvement screening test - ASSIST. É um instrumento composto de oito perguntas sobre o uso de nove classes de substâncias: tabaco, álcool, maconha, cocaína, estimulantes, sedativos, inalantes, alucinógenos e opiáceos (Group, 2002; Humeniuk, et al., 2008;).

A primeira questão aborda o consumo de cada substância ao longo da vida; entre a segunda e a quinta questão retrata a frequência, a necessidade de consumir determinada substância e fatores negativos decorrentes do uso; da sexta a oitava questão busca saber sobre preocupações de amigos e familiares a respeito do uso, se já houve tentativas de diminuição do consumo e se a pessoa já utilizou alguma substância injetável. As respostas recebem pontuações e sua somatória é de 0 a 39 pontos e possibilita a utilização desses pontos para a classificação dos indivíduos usuários conforme a severidade do padrão do uso, em: sem necessidade de intervenção, necessidade de intervenção breve e necessidade de intervenção intensiva (GranvilleGarcia, et al., 2009).

O terceiro foi um questionário sobre o conhecimento das consequências do uso de álcool, maconha e cocaína, desenvolvido pelo grupo de pesquisadores da Comissão Interamericana para o Controle e Abuso de Drogas (CICAD) em parceria com Centre for Addiction and Mental Health (CAMH), esse instrumento foi elaborado com base no Manual ASSIST e o Adverse Consequences of Substance Use Scale (ACSUS), onde permite mensurar se o conhecimento do estudante em alto, 50\% de acertos ou mais, ou baixo, menos de 50\% de acertos (Mann, Rootman, Shuggi \& Adlaf, 2006; Wright, et al., 2015; Souza, et al., 2018).

O questionário é composto por consequências sociais, psicológicas, biológicas e disctractors (consequências reais ou imaginárias não associadas às drogas), totalizando 14 itens para cada droga. Desse modo, 42 itens compõem o questionário, para assinalar verdadeiro ou falso, sendo o conhecimento para cada droga avaliado separadamente. 
Para apoio na coleta de dados, foram selecionados dois pesquisadores devidamente treinados para aplicação do questionário. A coleta foi conduzida no período de maio a dezembro de 2019. Inicialmente, o pesquisador obteve a lista de classes potenciais para participarem do estudo. Os responsáveis de cada turma foram contatados para obtenção de sua aprovação para administrar o estudo, durante um período de aula. No período acordado, em cada classe participante, os pesquisadores entraram na sala de aula apresentados pelo responsável, que, então, suspendeu a aula e saiu da sala.

O pesquisador explicou sobre o estudo aos presentes e os estudantes que aceitaram participar do estudo, receberam uma via do Termo de Consentimento Livre e Esclarecido (TCLE) e o instrumento de coleta de dados. O tempo médio para o preenchimento do questionário foi de 20 minutos. Os dados foram tabulados no Microsoft Excel e analisados através do software estatístico Statistical Package for Social Sciences (SPSS). Foram utilizadas estatísticas descritivas, como médias, desvios padrão, frequências, teste Qui-quadrado de Pearson e o Teste t de Student para análise dos dados. O nível alpha foi definido como $5 \%$.

\section{Resultados}

A amostra foi composta por 210 universitários, sendo 109 da rede privada e 101 da pública, desses, 71 cursavam o primeiro período e 139 estavam matriculados no último semestre. Dentre os pesquisados, a maioria era do sexo feminino (70,95\%), com faixa etária de 22 a 25 anos $(38,10 \%)$, moravam com a família $(82,86 \%)$, solteiros $(80 \%)$, com a renda per capita familiar de três a seis salários mínimos $(31,90 \%)$ e não trabalhavam $(78,10 \%)$ (Tabela 1).

Tabela 1. Distribuição dos universitários, segundo as variáveis sociodemográficas. (N=210).

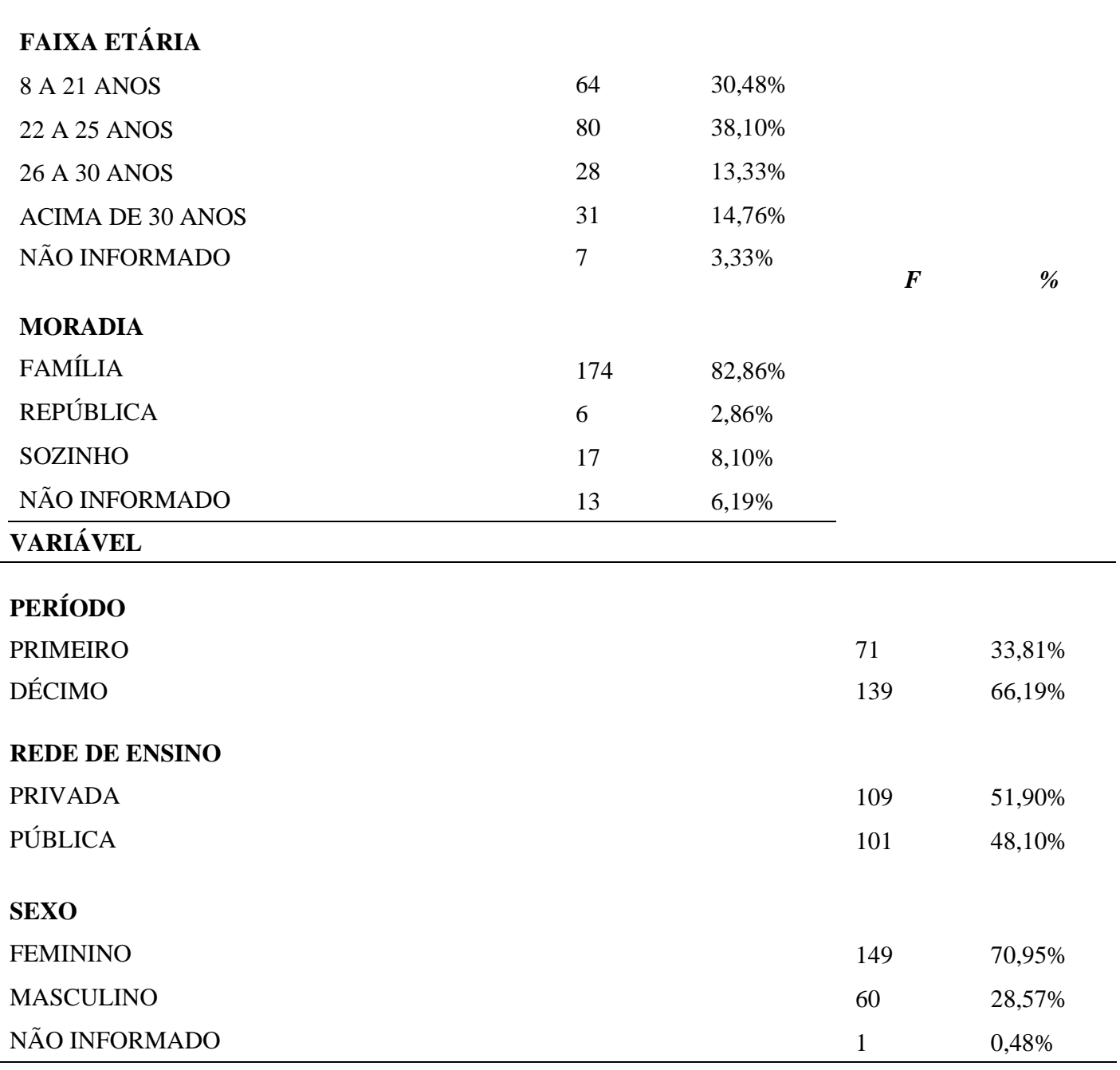


Fonte: Autores (2021).

Análises de frequência foram empregadas para investigar o uso na vida de drogas. Verificou-se que as drogas mais relatadas foram bebidas alcoólicas $(81,90 \%)$, derivados do tabaco $(25,24 \%)$ e a maconha $(24,76 \%)$. Cocaína/crack $(3,33 \%)$ e opióides $(1,43 \%)$ foram as drogas menos consumidas na vida (Gráfico 1).

Gráfico 1 - Porcentagem do uso de drogas, na vida, entre os universitários. (N=210).

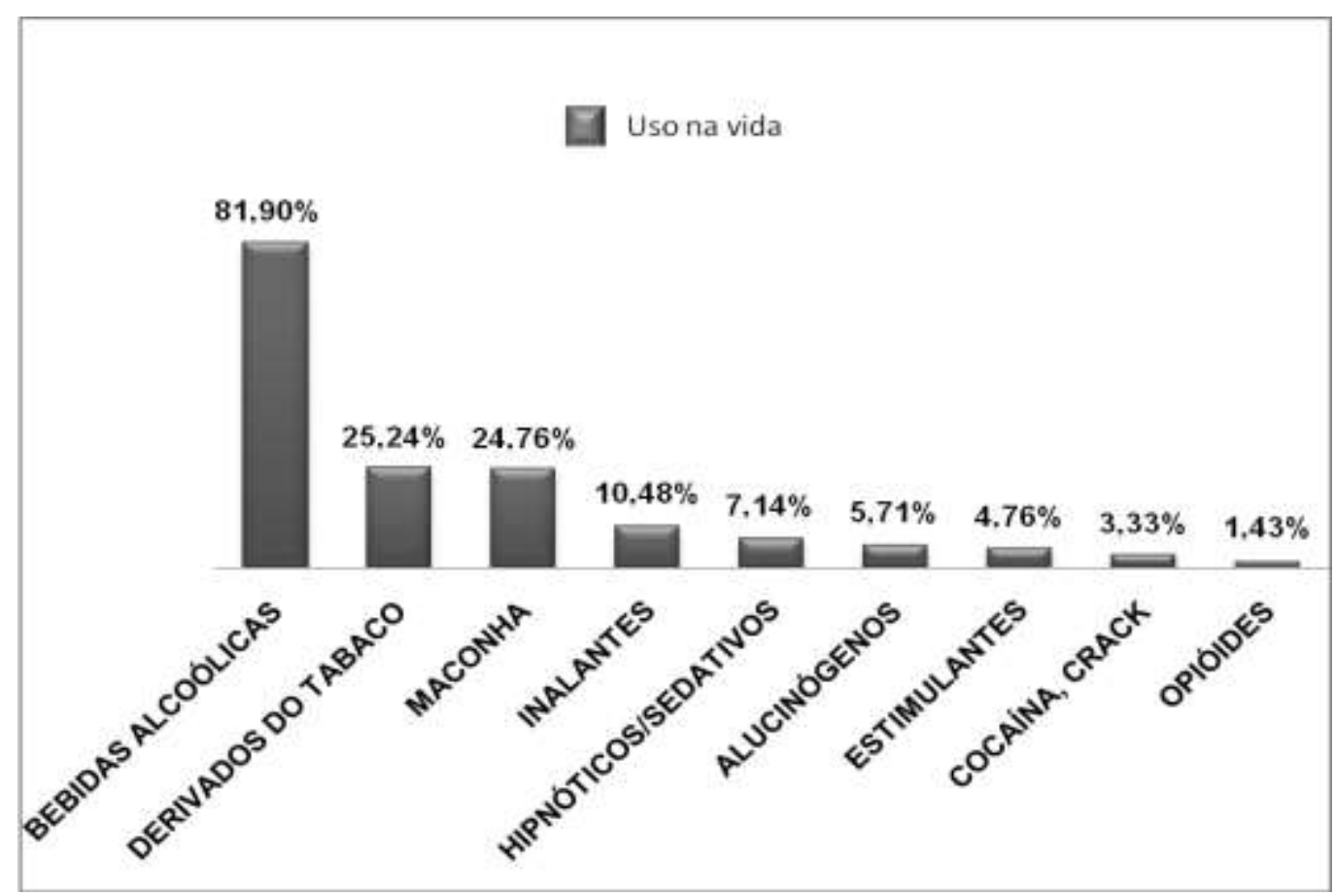

Fonte: Autores (2021).

Após as análises dos dados, observou-se que a maior prevalência do uso de drogas foi entre os universitários do décimo período, com exceção de álcool e opióides, predominante entre os estudantes do primeiro período. Já na análise com a rede de ensino, a maior prevalência do uso de drogas lícitas foi entre os estudantes da rede privada e as drogas ilícitas, entre os estudantes da rede pública. Quando avaliado o uso de drogas na vida em relação ao sexo, a maior prevalência foi no sexo masculino; apenas no uso de hipnótico-sedativos que no sexo feminino foi maior (Tabela 2). 
Tabela 2. Distribuição da frequência uso na vida de drogas por período, rede de ensino, sexo, faixa etária e moradia.
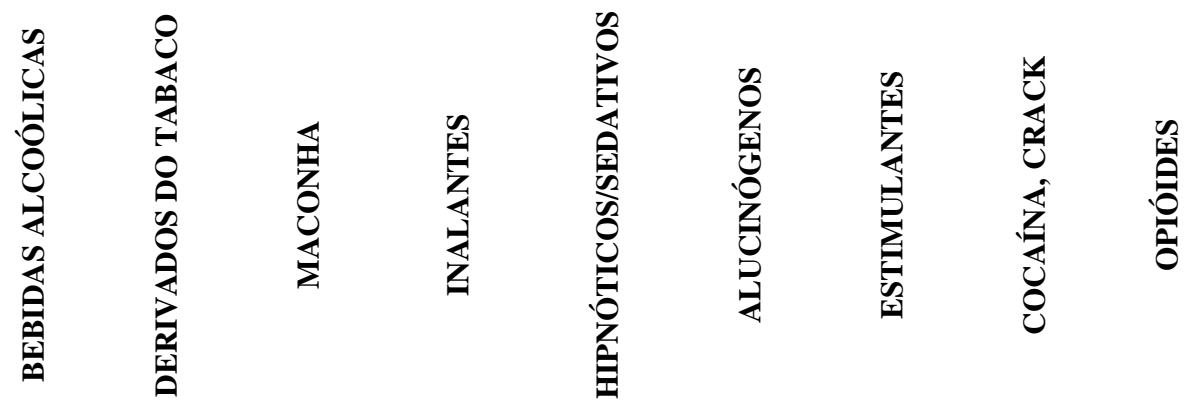

\section{PERÍODO}

PRIMEIRO

$85,92 \% \quad 23,94 \%$

$23,94 \% \quad 7,04 \%$

$5,63 \%$

$2,82 \%$

$4,23 \%$

$1,41 \%$

$2,82 \%$

DÉCIMO

$79,86 \% \quad 25,90 \%$

$25,18 \%$

$12,23 \%$

$7,91 \%$

$7,19 \%$

$5,04 \%$

$4,32 \%$

$0,72 \%$

\section{REDE DE ENSINO}

PRVADA

PÚBLICA

$81,65 \% \quad 25,69 \%$

$22,94 \%$

$9,17 \%$

$4,59 \% \quad 7,34 \%$

$4,59 \%$

$1,83 \%$

$0,00 \%$

$82,18 \% \quad 24,75 \%$

$26,73 \%$

$11,88 \%$

$9,90 \% \quad 3,96 \%$

$4,95 \%$

$4,95 \%$

$2,97 \%$

\section{SEXO}

FEMINIO

MASCULINO

$\begin{array}{ll}78,52 \% & 16,78 \% \\ 90,00 \% & 46,67 \%\end{array}$

$17,45 \%$

$6,71 \%$

$8,05 \%$

$2,01 \%$

$4,03 \%$

$0,67 \%$

$1,34 \%$

$43,33 \% \quad 20,00 \% \quad 5,00 \%$

$15,00 \%$

$6,67 \%$

$10,00 \%$

$1,67 \%$

\section{FAIXA ETÁRIA}

$\begin{array}{llllllllll}\text { 18 A 21 ANOS } & 85,94 \% & 20,31 \% & 21,88 \% & 9,38 \% & 7,81 \% & 4,69 \% & 3,13 \% & 1,56 \% & 3,13 \% \\ \text { 22 A 25 ANOS } & 83,75 \% & 30,00 \% & 32,50 \% & 12,50 \% & 8,75 \% & 6,25 \% & 6,25 \% & 5,00 \% & 1,25 \% \\ \text { 26 A 30 ANOS } & 75,00 \% & 32,14 \% & 28,57 \% & 7,14 \% & 3,57 \% & 3,57 \% & 7,14 \% & 7,14 \% & 0,00 \% \\ \begin{array}{l}\text { ACIMA DE 30 } \\ \text { ANOS }\end{array} & 80,65 \% & 19,35 \% & 12,90 \% & 12,90 \% & 6,45 \% & 9,68 \% & 3,23 \% & 0,00 \% & 0 \%\end{array}$

\section{MORADIA}

\begin{tabular}{llllllllll} 
FAMÍLIA & $82,18 \%$ & $22,99 \%$ & $20,69 \%$ & $9,77 \%$ & $6,90 \%$ & $4,02 \%$ & $4,60 \%$ & $1,72 \%$ & $1,72 \%$ \\
REPÚBLICA & $82,35 \%$ & $41,18 \%$ & $47,06 \%$ & $23,53 \%$ & $11,76 \%$ & $0 \%$ & $11,76 \%$ & $11,76 \%$ & $0,00 \%$ \\
SOZINHO & $84,62 \%$ & $30,77 \%$ & $53,85 \%$ & $7,69 \%$ & $7,69 \%$ & $38,46 \%$ & $0,00 \%$ & $15,38 \%$ & $0,00 \%$ \\
\hline
\end{tabular}

Fonte: Autores (2021).

Em relação à faixa etária, observou-se que a maior prevalência de álcool e opióides foi à faixa etária compreendida entre 18 e 21 anos, maconha e hipnóticos/sedativos entre 22 a 25 anos, derivados de tabaco, estimulantes e cocaína entre 26 a 30 anos, e inalantes e alucinógenos acima de 30 anos (Tabela 2).

Alguns universitários apresentaram necessidade de intervenção. Para intervenção breve os resultados variaram entre 16 universitários para bebidas alcoólicas, derivados de tabaco e maconha, derivados de tabaco, 5 para hipnóticos/sedativos e alucinógenos, 2 para inalantes, cocaína e opióides. Apenas 1 universitário apresentou a necessidade de tratamento mais intenso para o derivado de tabaco. 
Quanto às consequências do uso de álcool, maconha e cocaína, a maioria dos estudantes possuía um bom conhecimento, sendo que $96,67 \%$ obtiveram mais da metade de acertos em relação ao álcool, $87,14 \%$ para a maconha e 90,95\% para a cocaína. Foi calculado o escore para cada droga por meio da soma de um ponto para cada resposta correta, totalizando 14 pontos, onde as médias de acertos foram 12,55 para álcool, 11,22 para maconha e 11,57 para cocaína.

No teste de comparação das médias entre os universitários do primeiro e do último período e o conhecimento das consequências do uso de álcool, maconha e cocaína, não houve diferença significante; já entre as redes de ensino houve diferença significante para maconha $(\mathrm{p}=0,011)$ e cocaína $(\mathrm{p}=0,047)$, sendo que os alunos do último ano atingiram maiores escores (Tabela 3).

Tabela 3. Distribuição da média dos escores de acertos por período e rede de ensino segundo o conhecimento sobre as consequências do uso de álcool, maconha e cocaína.

\begin{tabular}{lcccccc}
\hline & \multicolumn{2}{c}{ PERÍODO } & & \multicolumn{3}{c}{ REDE DE ENSINO } \\
DROGA & $\begin{array}{c}\text { PRIMEIRO } \\
\text { Média (DP) }\end{array}$ & $\begin{array}{c}\text { DÉCIMO } \\
\text { Média (DP) }\end{array}$ & $* * p$ & $\begin{array}{c}\text { PRIVADA } \\
\text { Média (DP) }\end{array}$ & $\begin{array}{c}\text { PÚBLICA } \\
\text { Média (DP) }\end{array}$ & $* * p$ \\
\hline ÁLCOOL & $12,47(1,93)$ & $12,59(1,56)$ & 0,319 & $12,37(1,69)$ & $12,75(1,68)$ & 0,056 \\
MACONHA & $10,93(1,92)$ & $11,36(1,97)$ & 0,079 & $10,91(2,63)$ & $11,54(1,83)$ & 0,012 \\
COCAÍNA & $11,35(1,74)$ & $11,68(1,41)$ & 0,093 & $11,39(1,60)$ & $11,76(1,44)$ & 0,047 \\
\hline
\end{tabular}

* $p$ - derivado do Teste t de Student.

Fonte: Autores (2021).

Na relação entre o consumo de álcool, maconha e cocaína na vida com o conhecimento sobre as consequências do uso dessas substâncias, os resultados mostraram não haver associação estatisticamente significativa. Dos 172 dos universitários que já fizeram uso do álcool na vida, 97,09\% tiveram escore acima da média para o conhecimento das consequências do uso do álcool. Já no que diz respeito à maconha, o maior percentual de acertos $(97,47 \%)$ foi entre os 158 universitários que não fizeram uso de tal substância. Em relação à cocaína, 100\% dos universitários que consumiram cocaína na vida tiveram escores acima da média, já com os que não consumiram, 89,69\% tiveram escores acima da média (Tabela 4).

Tabela 4. - Distribuição dos estudantes de acordo com o consumo na vida de álcool, maconha e cocaína segundo o conhecimento sobre as consequências do uso dessas substâncias.

\begin{tabular}{|c|c|c|c|c|c|c|c|c|c|}
\hline & \multicolumn{3}{|c|}{ ÁLCOOL } & \multicolumn{3}{|c|}{ MACONHA } & \multicolumn{3}{|c|}{ COCAÍNA } \\
\hline & $\begin{array}{l}\text { Acima } \\
\text { Média }\end{array}$ & $\begin{array}{l}\text { Abaixo } \\
\text { Média }\end{array}$ & & $\begin{array}{l}\text { Acima } \\
\text { Média }\end{array}$ & $\begin{array}{l}\text { Abaixo } \\
\text { Média }\end{array}$ & & $\begin{array}{l}\text { Acima } \\
\text { Média }\end{array}$ & $\begin{array}{c}\text { Abaixo } \\
\text { Média }\end{array}$ & \\
\hline Uso de drogas & $(\%)$ & $(\%)$ & $p^{*}$ & $(\%)$ & $(\%)$ & $p^{*}$ & $(\%)$ & $(\%)$ & $p^{*}$ \\
\hline \multirow[t]{2}{*}{ SIM } & $97,09 \%$ & $2,91 \%$ & & $94,23 \%$ & $5,77 \%$ & & $100,00 \%$ & $0 \%$ & \\
\hline & & & 0,463 & & & 0,259 & & & 0,396 \\
\hline NÃO & $94,74 \%$ & $5,26 \%$ & & $97,47 \%$ & $2,53 \%$ & & $90,64 \%$ & $9,36 \%$ & \\
\hline
\end{tabular}

$p^{*}$ - derivado do teste qui-quadrado de Pearson.

Fontes: Autores (2021). 


\section{Discussão}

A prevalência de uso drogas é alta entre os estudantes universitários (Kerr-Corrêa, Andrade, Bassit \& Boccuto, 1999; de Medeiros, et al., 2012; Ramalho, 2019; Mendonça, 2022). Esses dados demonstram a preocupação da comunidade mundial quanto a esse uso abusivo de drogas entre universitários (Eckschmidt, Andrade \& Oliveira, 2013; Mustafá, et al., 2019; Gonçalves, Fava, Alves \& Dázio, 2019). Reduzir esse consumo abusivo provavelmente será benéfico para a sociedade como um todo.

O índice de prevalência de álcool (82,17\%), do presente estudo foi semelhante ao de Mendonça (2020), em que entre 1.147 universitários do primeiro e do penúltimo período dos cursos de Medicina, Odontologia, Enfermagem, Fisioterapia e Nutrição de duas universidades, sendo uma pública e outra privada de Aracaju/SE, o qual teve a prevalência do consumo de bebida alcoólica na vida dos universitários de 80,7\%. Contudo, em um estudo, internacional entre estudantes mexicanos de medicina, foi observada uma redução do uso de álcool ao avaliarem esse padrão de consumo, o qual detectou uma prevalência de 71,9\% de uso de álcool (Sanchez, Oliveira, Ribeiro, Nappo, 2010).

Mendonça (2020) afirma que a elevada prevalência de consumo de álcool entre os universitários, se dá pela ampla aceitação social, bem como a existência de anúncios comerciais e letras de músicas direcionadas ao público jovem que incentivam o uso do álcool e omitem os seus malefícios.

Granville-Garcia et al. (2009) mostraram que houve prevalência de 7,1\% do tabagismo entre estudantes do curso de Odontologia da Universidade Estadual da Paraíba. No presente estudo, observou-se uma prevalência do uso de derivados de tabaco de $24,35 \%$. No que concerne à área de saúde, a pesquisa de Rodrigues et al. (2008), mostrou que é elevada a taxa de fumantes entre os cirurgiões-dentistas.

No que tange ao uso de maconha, a prevalência foi de $24,76 \%$, sendo esse dado superior aos encontrados por Teixeira et al. (2010), ao avaliar o uso desse entorpecente entre graduandos de odontologia, cuja prevalência foi de $13,2 \%$. O uso da maconha no âmbito universitário pode estar relacionado à busca de diferentes estímulos, dentre eles a fuga de problemas, podendo ser pessoais, financeiros, emocionais ou psicológicos. Podem ser considerados como gatilhos para o uso da maconha entre universitários a pressão imposta por grupos sociais, pré-disposição genética, problemas de personalidade, baixa autoestima, falta de estimo devida, procura de sensações de prazer e a curiosidade (Sanchez, Oliveira, Ribeiro, Nappo, 2010; Cruz, 2017; Alves \& MacRae, 2019).

A prevalência do uso de cocaína, anfetaminas, êxtase, inalantes, hipnóticos, sedativos, alucinógenos e opióides observada no presente estudo foi semelhante aos resultados encontrados em outros estudos, variando entre $1,43 \%$ a $10,48 \%$ de uso na vida por substância (Teixeira, Souza, Buaiz \& Siqueira, 2010; de Medeiros, et al., 2012; Ramalho, 2019.

Observa-se que o décimo período teve a maior prevalência de uso de substâncias, enquanto que o consumo de álcool foi maior entre os estudantes do primeiro. Esse resultado corrobora com Castaldelli-Maia et al. (2013), em que essa prevalência está relacionada ao estresse de final do curso, sobrecarga de estágios e com mudanças de hábitos.

Entre as redes de ensino, foi constatada uma maior prevalência do uso de drogas lícitas na rede privada enquanto que na rede pública, o maior consumo foi de drogas ilícitas. Houve um maior abuso dessas substâncias entre os estudantes que residiam em repúblicas ou sozinhos. A maioria dos estudantes de universidades públicas residiam longe dos seus familiares, os tornando mais expostos ao consumo (Mustafá, et al., 2019). Com isso a presença da família pode ser considerada um fator de proteção (Gonçalves, Fava, Alves \& Dázio, 2019).

Ao analisar por sexo, o consumo de drogas, com exceção de hipnóticos-sedativos, foi mais prevalente no sexo masculino, como já documentado na literatura (Stempliuk, et al., 2005, Teixeira, Souza, Buaiz \& Siqueira, 2010). Esse predomínio do consumo em homens pode estar ligado às construções socioculturais de gênero (Dázio, Zago, Fava, 2016). 
Já em relação ao uso de hipnóticos/sedativos, houve um abuso maior por parte do sexo feminino, o que não é condizente em estudos realizados por Teixeira et al. (2012), e Mustafá et al. (2019). No entanto, este resultado pode se dá por diferenças locais, e devem ser mais bem investigados.

Em relação à faixa etária, foi observada uma maior prevalência do uso de drogas entre os estudantes de 22 a 25 anos. Corroborando com os dados de Gonçalves et al. (2019) e Ramalho et al. (2019). Esse resultado pode ser multifatorial e pode estar relacionada à fase autônoma e independente do grupo estudado.

Na avaliação do uso de drogas com a necessidade de intervenção, a maioria dos estudantes não necessitava que fossem realizadas intervenções. Resultados encontrados por Medeiros et al. (2012), revelaram uma prevalência superior a encontrada em nossa pesquisa. É possível que a severidade do padrão do uso esteja ligada à faixa etária dos universitários estudados, no qual o abuso dessas substâncias se faz mais presente com o passar do tempo.

Quanto ao conhecimento acerca das consequências do uso de droga, os resultados mostraram que a maioria dos universitários apresentou níveis de conhecimento acima da média. Assim, o bom nível de conhecimento apresentado sugere que estes saberes não foram construídos necessariamente no âmbito universitário, mas provavelmente adquiridos ao longo de suas experiências de vida ou por meio de estratégias de prevenção mediadas por meios de comunicação ou escolas de educação básica (Souza, et al., 2018).

$\mathrm{Na}$ análise da correlação dos conhecimentos das consequências com o período em que os estudantes estavam matriculados, os estudantes do décimo período obtiveram as maiores médias, apresentando diferenças de 0,12 para álcool, 0,43 para maconha e 0,33 para cocaína, para os estudantes do primeiro período. Esperava-se dos alunos do último ano o conhecimento mais específico, amplo e adequado sobre as consequências dessas drogas, contudo, a diferença não foi significante. Importante destacar que os estudantes do primeiro período já iniciam a graduação com o conhecimento empírico sobre a temática, diante da ausência de diferença estatística importante entre os grupos (Souza, et al., 2018).

Com relação à rede de ensino, observou-se que os estudantes da pública apresentaram médias superiores aos estudantes da privada. Nesse aspecto, a diferença foi significante para o entendimento das consequências do uso de maconha e cocaína, sugerindo que o ambiente de ensino pode interferir na construção de tal conhecimento. Em contrapartida, cabe a inquirir se este fato reflete no ambiente de ensino ou advém de um menor interesse dos usuários de maconha e cocaína em buscar informações sobre esta temática.

Foi possível observar que os universitários usuários de álcool e cocaína apresentaram um percentual de acerto maior que os universitários que não as consomem, mostrando que o conhecimento sobre as consequências do uso não é necessariamente um fator proteção para o uso dessas substâncias. Contudo, ao correlacionar o uso de maconha e o conhecimento sobre suas consequências, os universitários que referiram não ter feito uso de maconha obtiveram um melhor nível de conhecimento, corroborando com resultados de Souza (2018), que sugerem que a informação pode ser um fator de proteção.

O uso de drogas em suas dimensões sociais, culturais, econômica, familiar e individual é uma questão complexa, e por isso faz-se necessário a realização de estudos mais representativos para o conhecimento profundo desta problemática, no intuito de que se possa proporcionar eficácia nas intervenções de prevenção e controle do uso indevido desta droga (Eckschmidt, Andrade \& Oliveira, 2013).

\section{Conclusão}

$\mathrm{Na}$ cidade de Recife e região metropolitana, o consumo de drogas entre os estudantes universitários de odontologia apresentou prevalências elevadas. O consumo foi mais prevalente entre o sexo masculino, e universitários do décimo período, da rede pública, na faixa etária de 22 a acima de 25 anos, que residiam em repúblicas ou sozinhos. A maioria dos universitários 
obteve mais da metade de acertos na análise das consequências do uso do álcool, maconha e cocaína, considerado um bom conhecimento. Foi constatado que o conhecimento das consequências não foi um fator de proteção para o uso das mesmas. Novos estudos são necessários para detalhar esse consumo de drogas entres os universitários e identificar a causa da associação ente o uso de substâncias psicoativas e o conhecimento das conseqüências de seu uso.

\section{Referências}

Almeida, N. D. (2017). Uso de álcool, tabaco e drogas por jovens e adultos da cidade de Recife. Psicologia argumento, 29(66). Disponível a partir de https://periodicos.pucpr.br/index.php/psicologiaargumento/article/view/20285

Alves, Y. D. D., \& MacRae, E. (2019). Uma abordagem teórica sobre o contexto social do uso de drogas. Revista TOMO, (34), 81-114. $0.21669 /$ tomo.v0i34.9850

Arora, A., Kannan, S., Gowri, S., Choudhary, S., Sudarasanan, S., \& Khosla, P. P (2016). Abuso de substâncias entre os estudantes de graduação em medicina em um país em desenvolvimento. The Indian Journal of Medical Research, 143 (1), 101-103. 10.4103/0971-5916.178617

Bublitz, S., Freitas, E., Kirchhof, R., Lopes, L., \& Guido, L. (2013). Estressores entre acadêmicos de enfermagem de uma universidade pública [Stressors among nursing students at a public university] [Estresores entre estudiantes de enfermería de uma universidad pública]. Revista Enfermagem UERJ, 20(6), 739-745. Disponível a partir de https://www.e-publicacoes.uerj.br/index.php/enfermagemuerj/article/view/5992

Brasil. Presidência da República. Secretaria Nacional de Políticas sobre Drogas. (2010). I Levantamento Nacional sobre o Uso de Álcool, Tabaco e Outras Drogas entre Universitários das 27 Capitais Brasileiras / Secretaria Nacional de Políticas sobre Drogas; GREA/IPQ-HCFMUSP; organizadores Arthur Guerra de Andrade, Paulina do Carmo Arruda Vieira Duarte, Lúcio Garcia de Oliveira. - Brasília: SENAD, 284 p. https://www.mds.gov.br/webarquivos/arquivo/cuidados_prevencao_drogas/obid/publicacoes/Livros/I\%20Levantamento\%20Nacional\%20Universit\%C3\%A1r ios\%20-\%202010.pdf

Canoletti, B., \& Soares, C. B. (2005). Programas de prevenção ao consumo de drogas no Brasil: uma análise da produção científica de 1991 a 2001. InterfaceComunicação, Saúde, Educação, 9, 115-129. doi:10.1590/S1414-32832005000100010

Castaldelli-Maia, J. M., Martins, S. S., De Oliveira, L. G., Van Laar, M., De Andrade, A. G., \& Nicastri, S. (2014). Use transition between illegal drugs among Brazilian university students. Social psychiatry and psychiatric epidemiology, 49(3), 385-394. https://doi.org/10.1007/s00127-013-0748-1

Cruz, M. J. B. (2013). Uso de drogas entre os jovens e adolescentes-da curiosidade à dependência. Universidade Federal de Minas Gerais. Faculdade de Medicina. Núcleo de Educação em Saúde Coletiva. Diamantina. 33f. Monografia (Especialização em Atenção Básica em Saúde da Família). Disponível a partir de https://www.nescon.medicina.ufmg.br/biblioteca/registro/Uso_de_drogas_entre_os_jovens_e_adolescentes__da_curiosidade_a_dependencia/461

Dázio, E. M. R., Zago, M. M. F., \& Fava, S. M. C. L. (2016). Uso de álcool e outras drogas entre universitários do sexo masculino e seus significados. Revista da Escola de Enfermagem da USP, 50, 785-791. 10.1590/S0080-623420160000600011

de Barros Junqueira, M. A., Rassool, G. H., dos Santos, M. A., \& Pillon, S. C. (2015). The Impact of an Educational Program in Brief Interventions for Alcohol Problems on Undergraduate Nursing Students: A Brazilian Context. Journal of Addictions Nursing, 26(3), 129-135. 10.1097/jan.0000000000000086

de Medeiros, S. B., Rediess, S. V., Hauck Filho, N., Martins, M. I. M., \& Mazoni, C. G. (2012). Prevalência do uso de drogas entre acadêmicos de uma universidade particular do sul do Brasil. Aletheia, (38-39). 10.29327/226091

Eckschmidt, F., Andrade, A. G. D., \& Oliveira, L. G. D. (2013). Comparison of drug use between Brazilian and American college students and young Brazilian general population. Jornal Brasileiro de Psiquiatria, 62(3), 199-207. 10.1590/S0047-20852013000300004

Estrela, C. (2018). Metodologia Científica: Ciência, Ensino, Pesquisa. Editora Artes Médicas.

Gonçalves, J. S., Fava, S. M. C. L., Alves, A. C., \& Dázio, E. M. R. (2019). Reflexões acerca do panorama de consumo de álcool e/ou outras drogas entre estudantes universitários. Revista de Enfermagem do Centro-Oeste Mineiro, 9. 10.19175/recom.v9i0.2594

Granville-Garcia, A. F., Branco, A. C. L., Sarmento, D. J. de S., Cavalcanti, A. L., D’Avila, S., \& Menezes, V. A. de. (2010). Tabagismo e fatores associados entre acadêmicos de odontologia. Revista Da Faculdade De Odontologia - UPF, 14(2). 10.5335/rfo.v14i2.720

Group, W. A. W. (2002). The alcohol, smoking and substance involvement screening test (ASSIST): development, reliability and feasibility. Addiction, 97(9), 1183-1194. 10.1046/j.1360-0443.2002.00185.x

Humeniuk, R., Ali, R., Babor, T. F., Farrell, M., Formigoni, M. L., Jittiwutikarn, J., \& Simon, S. (2008). Validation of the alcohol, smoking and substance involvement screening test (ASSIST). Addiction, 103(6), 1039-1047. 10.1111/j.1360-0443.2007.02114.x

Kerr-Corrêa, F., Andrade, A. G. D., Bassit, A. Z., \& Boccuto, N. M. V. F. (1999). Uso de álcool e drogas por estudantes de medicina da Unesp. Brazilian Journal of Psychiatry, 21, 95-100. 10.1590/S1516-44461999000200005.

Mann, R. E., Rootman, D. B., Shuggi, R., \& Adlaf, E. (2006). Assessing consequences of alcohol and drug abuse in a drinking driving population. Drugs: education, prevention and policy, 13(4), 313-326. 10.1080/09687630600624634

Mendonça, A. K. R. H. (2017). Padrão de consumo alcoólico e prática do binge drinking entre universitários da área de saúde. Universidade Tiradentes. Dissertação de Mestrado (Saúde e Ambiente) - Universidade Tiradentes. Programa de Pós-Graduação em Saúde e Ambiente. Aracaju. Disponível a partir de https://mestrados.unit.br/psa/wp content/uploads/sites/6/2017/03/DissertacaoAnaKarinaRHMendonca2017.pdf 
Mustafá, A. M. M., Gomides, M. M., Costa, J. L., Pires, A. T., \& Silva, Â. M. (2019). O uso de álcool e drogas por estudantes universitários brasileiros: um estudo teórico. Facit Business and Technology Journal, 1(10). https://jnt1.websiteseguro.com/index.php/JNT/article/viewFile/408/333

Pechansky, F., Szobot, C. M., \& Scivoletto, S. (2004). Uso de álcool entre adolescentes: conceitos, características epidemiológicas e fatores etiopatogênicos. Brazilian Journal of Psychiatry, 26, 14-17. 10.1590/S1516-44462004000500005

Pillon, S. C., O'brien, B., \& Chavez, K. A. P. (2005). The relationship between drugs use and risk behaviors in Brazilian university students. Revista LatinoAmericana de Enfermagem, 13(spe2), 1169-1176. 10.1590/S0104-11692005000800011

Puig-Lagunes, Ángel A., Nolasco, Ángel P., Mendez, L. E. S., Álvarez, J. E. V., \& Pillon, S. C. (2019). Consumo de álcool entre estudantes mexicanos de bacharelado. SMAD Revista Eletrônica Saúde Mental Álcool E Drogas (Edição Em Português), 15(3), 1-9. 10.11606/issn.1806-6976.smad.2019.151938

Ramalho, A. A. (2019). O consumo de álcool e outras drogas entre alunos ingressantes em cursos da área de saúde. Trabalho de Conclusão de Curso (Graduação em Enfermagem) - Faculdade de Ciências da Educação e Saúde, Centro Universitário de Brasília, Brasília, Disponível a partir de https://repositorio.uniceub.br/jspui/handle/prefix/13622.

Rodrigues, G. A., Galvão, V., \& Viegas, C. A. D. A. (2008). Prevalência do tabagismo entre dentistas do Distrito Federal. Jornal Brasileiro de Pneumologia, 34, 288-293. https://www.scielo.br/j/jbpneu/a/6GGSnrwjCrNjNb9DPd5gSBn/?format=pdf\&lang=pt

Vargas, D. D., \& Duarte, F. A. B. (2011). Enfermeiros dos centros de atenção psicossocial em álcool e drogas (Caps ad): a formação e a busca pelo conhecimento específico da área. Texto \& Contexto-Enfermagem, 20, 119-126. 10.1590/S0104-07072011000100014

Vieira, P. C., Aerts, D. R. G. D. C., Freddo, S. L., Bittencourt, A., \& Monteiro, L. (2008). Alcohol, tobacco, and other drug use by teenage students in a city in Southern Brazil. Cadernos de saude publica, 24(11), 2487-2498. 10.1590/S0102-311X2008001100004

Silva, L. V., Malbergier, A., Stempliuk, V. D. A., \& Andrade, A. G. D. (2006). Fatores associados ao consumo de álcool e drogas entre estudantes universitários. Revista de Saúde Pública, 40, 280-288. 10.1590/S0034-89102006000200014

Stempliuk, V. D. A., Barroso, L. P., Andrade, A. G. D., Nicastri, S., \& Malbergier, A. (2005). Comparative study of drug use among undergraduate students at the University of São Paulo: São Paulo campus in 1996 and 2001. Brazilian Journal of Psychiatry, 27, 185-193. 10.1590/S1516-44462005000300006

Souza, J. D., Ornella, K. P., Almeida, L. Y. D., Domingos, S. G. D. A., Andrade, L. S. D., \& Zanetti, A. C. G. (2018). Consumo de drogas e conhecimento sobre suas consequências entre estudantes de graduação em enfermagem. Texto \& Contexto-Enfermagem, 27. 10.1590/0104-070720180005540016

Teixeira, R. F., Souza, R. S. D., Buaiz, V., \& Siqueira, M. M. D. (2010). Psychoactive substance use among Espírito Santo Federal University odontology students. Ciencia \& saude coletiva, 15(3), 655-662. 10.1590/S1413-81232010000300007

Wright, M. D. G. M., Cumsille, F., Padilha, M. I., Ventura, C. A., Sapag, J., Brands, B. \& Khenti, A. (2015). International research capacity building program for health related professionals to study the drug phenomenon in Latin America and the Caribbean. Texto \& Contexto-Enfermagem, 24, 17-25. 10.1590/010407072015001010014 